\title{
Article
}

\section{Three new pulsating sdB stars discovered with SuperWASP}

\author{
Holdsworth, Daniel Luke, Ostensen, Roy H, Smalley, Barry and \\ Telting, John H \\ Available at http://clok.uclan.ac.uk/17051/ \\ Holdsworth, Daniel Luke ORCID: 0000-0003-2002-896X, Ostensen, Roy H, \\ Smalley, Barry and Telting, John H (2017) Three new pulsating sdB stars \\ discovered with SuperWASP. Monthly Notices of the Royal Astronomical \\ Society, 466 (4). pp. 5020-5032. ISSN 0035-8711
}

It is advisable to refer to the publisher's version if you intend to cite from the work. http://dx.doi.org/10.1093/mnras/stx077

For more information about UCLan's research in this area go to http://www.uclan.ac.uk/researchgroups/ and search for <name of research Group>.

For information about Research generally at UCLan please go to http://www.uclan.ac.uk/research/

All outputs in CLoK are protected by Intellectual Property Rights law, including Copyright law. Copyright, IPR and Moral Rights for the works on this site are retained by the individual authors and/or other copyright owners. Terms and conditions for use of this material are defined in the policies page.

\section{CLoK}

Central Lancashire online Knowledge www.clok.uclan.ac.uk

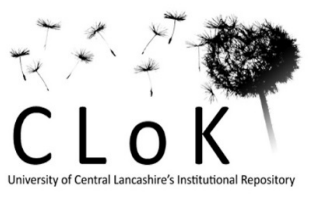




\title{
Three new pulsating sdB stars discovered with SuperWASP
}

\author{
Daniel L. Holdsworth, ${ }^{1,2 \star}$ Roy H. Østensen, ${ }^{3}$ Barry Smalley ${ }^{2}$ and John H. Telting ${ }^{4}$ \\ ${ }^{1}$ Jeremiah Horrocks Institute, University of Central Lancashire, Preston PR1 2HE, UK \\ ${ }^{2}$ Astrophysics Group, Keele University, Staffordshire ST5 5BG, UK \\ ${ }^{3}$ Department of Physics, Astronomy, and Materials Science, Missouri State University, Springfield, MO 65804, USA \\ ${ }^{4}$ Nordic Optical Telescope, Rambla José Ana Fernández Pérez 7, E-38711 Breña Baja, Spain
}

Accepted 2017 January 10. Received 2017 January 10; in original form 2016 November 10

\begin{abstract}
We present an analysis of three new pulsating subdwarf B stars discovered in the Super Wide Angle Search for Planets archive. Two of the stars, J1938+5609 and J0902-0720, are pmode pulsators; $\mathrm{J} 1938+5609$ shows a pulsation at $231.62 \mathrm{~d}^{-1}(P=373 \mathrm{~s} ; 2681 \mu \mathrm{Hz})$ with an amplitude of 4 mmag, whereas J0902-0720 pulsates at frequencies $636.74(P=136 \mathrm{~s}$; $7370 \mu \mathrm{Hz})$ and $615.34 \mathrm{~d}^{-1}(P=140 \mathrm{~s} ; 7122 \mu \mathrm{Hz})$, with amplitudes 7.27 and $1.53 \mathrm{mmag}$, respectively. The third star, J2344-3427, is a hybrid pulsator with a p-mode frequency at $223.16 \mathrm{~d}^{-1}(P=387 \mathrm{~s} ; 2583 \mu \mathrm{Hz})$ and a corresponding amplitude of $1.5 \mathrm{mmag}$, and g modes in the frequency range $8.68-28.56 \mathrm{~d}^{-1}(P=3025-9954 \mathrm{~s} ; 100-331 \mu \mathrm{Hz})$ and amplitudes between 0.76 and 1.17 mmag. Spectroscopic results place J1938+5609 and J2344-3427 among the long-period or hybrid pulsators, suggesting there may be further modes in these stars below our detection limits, with J0902-0720 placed firmly amongst the p-mode pulsators.
\end{abstract}

Key words: asteroseismology -techniques: photometric-stars: oscillations-subdwarfs.

\section{INTRODUCTION}

The hot subdwarf B (sdB) stars are evolved low-mass $\left(M \lesssim 0.5 \mathrm{M}_{\odot}\right)$ stars that consist of helium burning cores and a thin hydrogen atmosphere that is unable to support H-shell burning (Heber et al. 1984; Heber 1986). They are found on the extreme horizontal branch (EHB) with temperatures between about 22000 and $40000 \mathrm{~K}$, and surface gravities between values of $\log g=5.0-6.2$. The progenitors to these stars, main-sequence stars with masses $\lesssim 2 \mathrm{M}_{\odot}$, have undergone a core helium flash and made their way to the red giant branch. Here they retain approximately 1 per cent of their hydrogen mass. As a result of this low hydrogen mass, after core helium exhaustion, the stars move directly to the white dwarf (WD) cooling track, rather than moving to the asymptotic giant branch phase. The lifetime for the EHB stars is short: to move from the zero-age EHB to the terminal-age EHB takes between 100 and 150 Myr.

The formation of sdB stars is still somewhat of a mystery, in particular the mechanism that causes only a small amount of $\mathrm{H}$ to remain in an envelope while the He core mass is of the order of $0.5 \mathrm{M}_{\odot}$. Mengel, Norris \& Gross (1976) suggested mass-loss in Roche lobe filling binaries could account for the resulting EHB star, while Webbink (1984) suggested WD mergers as a possible progenitor. More recently, Maxted et al. (2001, 2002) found evidence that a large number of $\mathrm{sdB}$ stars are found in binaries, adding weight to the binary formation theory. Following from that discovery,

^E-mail: dlholdsworth@uclan.ac.uk several mechanisms have been proposed for the resulting low-mass H envelope of the EHB stars (Han et al. 2002, 2003). For a thorough overview of the sdB stars as a whole, see Heber $(2009,2016)$.

There exists a subgroup of the $\mathrm{sdB}$ stars that show light variations of the order of seconds to hours: the subdwarf B variable (sdBV) stars. The prototype for this class, EC 14026-2647, was observed by Kilkenny et al. (1997) as part of the Edinburgh-Cape (EC) survey (Stobie et al. 1997b). They found the star to be pulsating with a period of $144 \mathrm{~s}\left(v=600 \mathrm{~d}^{-1} ; 6944 \mu \mathrm{Hz}\right)$ and an amplitude of 12 mmag.

As the number of sdBV stars grew, it became clear that two types of pulsators exist. The initial set of sdBV stars showed very short period pulsations, which were identified as pressure-mode (p-mode) oscillations by Charpinet et al. (1997). These modes are driven in the envelopes of the stars. A later discovery by Green et al. (2003) showed a longer period variation in the sdB star PG $1716+426$. The period in this, and other stars, was of the order 10 times longer than those previously seen in the sdB stars, and there was a distinct difference between the temperatures of the initial group of pulsators and this new one. These observations led to the conclusion that the newly identified sdBVs were gravity-mode (g-mode) pulsators (Fontaine et al. 2003). Since the first discovery of variability in the subdwarf stars, $110 \mathrm{sdBV}$, sdOBV and sdOV stars have been identified. Table 1 lists the known sdBV and sdOV stars (hereafter sdV when referred to jointly).

As is seen with stars on the main sequence, there is a final subset of sdV stars that show both low- and high-frequency pulsations. These hybrid pulsators (also called DW Lyn stars after their prototype), 


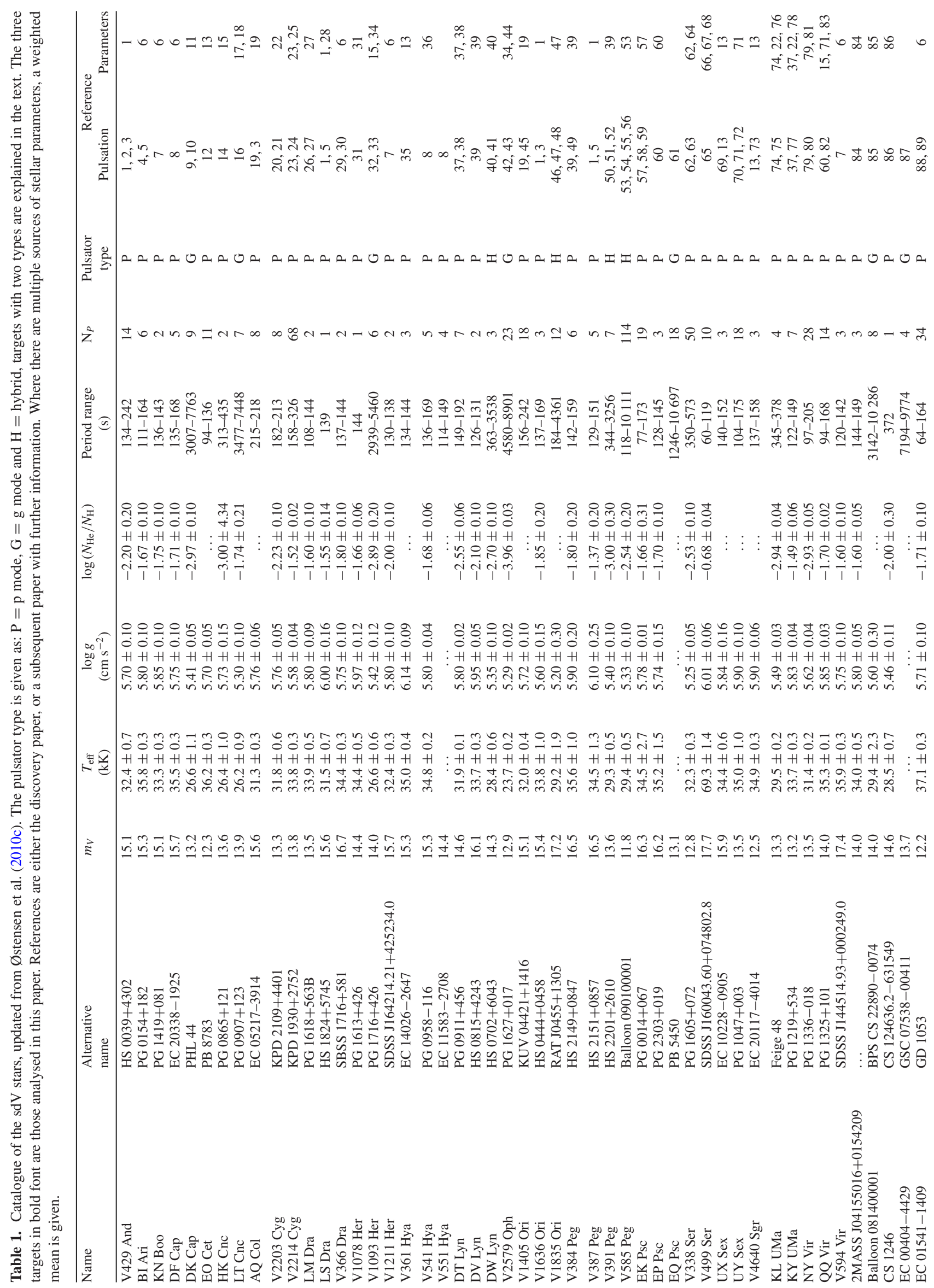




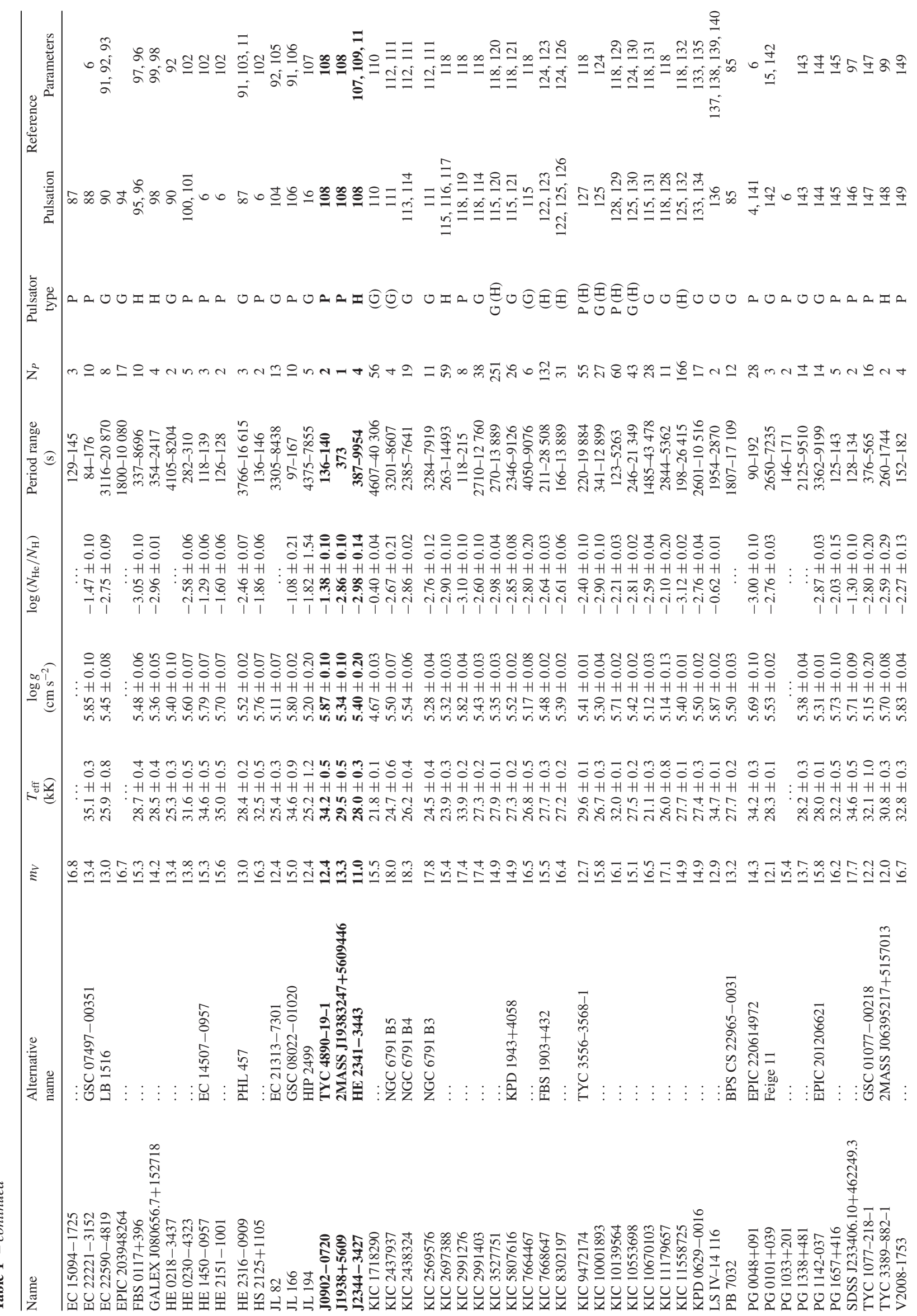




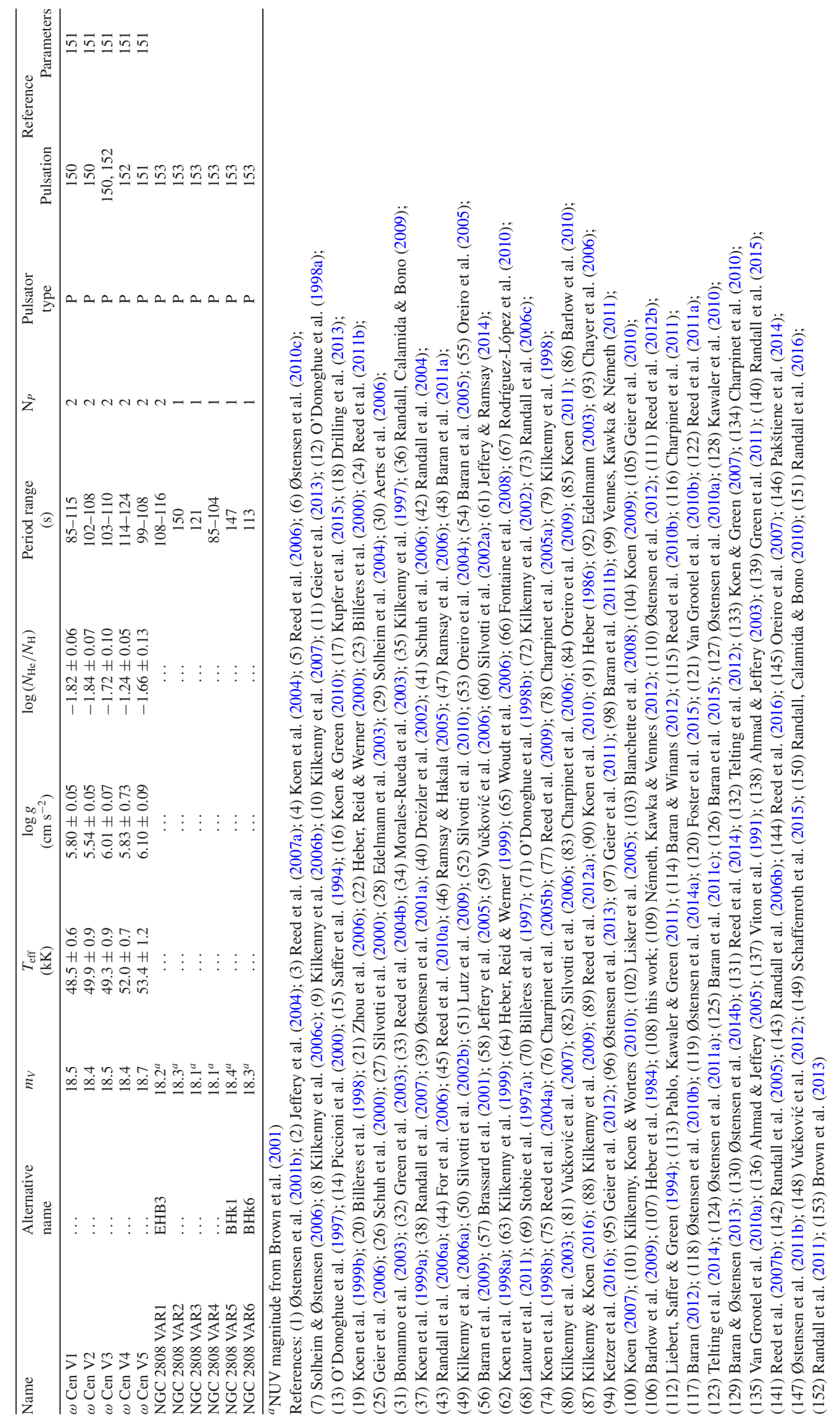


first seen by Schuh et al. (2006), provide the ideal laboratory to test the internal structure of the subdwarf stars. The presence of both $\mathrm{p}$ and $g$ modes in a single star allows for detailed asteroseismological modelling of the interior as the different modes probe varying layers in the star. For example, to explain the presence of both modes in a single star, Jeffery \& Saio (2006) were able to extend the instability strip of the sdBV stars, so that the p- and g-mode regions overlapped, by including nickel opacities in their calculations.

The original differences in pulsation frequency that were used to identify p-mode, g-mode and hybrid pulsators are not robust enough when presented with precise space-based observations. Therefore, in Table 1, we class the spaced-based observations as if they were observed from the ground, with a detection limit of 1 mmag. If there are other pulsations below this limit, a classification of ' $(\mathrm{H})$ ' is given to indicate that the star is a hybrid pulsator at the detection limit of the Kepler satellite. This notation is also used in the cases where all pulsations are below the ground-based detection limit, i.e. in the cases where a classification of ' $(\mathrm{G})$ ' is given.

The top panel of Fig. 1 shows all the known sdV stars, which have values of $T_{\text {eff }}$ and $\log g$ in Table 1, in the $T_{\text {eff }}-\log g$ plane. Two groups form in the diagram, the blue points that represent the g-mode pulsators, and the red points that represent the p-mode pulsators. The g-mode pulsators have systematically lower temperatures and surface gravities than their p-mode counterparts. The hybrid stars, as one would expect, straddle the two groups, and are shown in black. One star of particular note is the g-mode pulsator LS IV14 116, the blue dot amongst the red. This star shows an extremely peculiar abundance pattern and is thought to belong to the halo population (Randall et al. 2015). LS IV-14 116 is also an outlier in the middle and bottom panels of Fig. 1 where the abnormal helium abundance is obvious and it is the only high surface gravity g-mode pulsator. Models of this star, using the opacity mechanism, cannot explain the pulsations given the derived stellar parameters. Despite considerable effort, this star remains a mystery (e.g. Green et al. 2011; Miller Bertolami, Córsico \& Althaus 2011; Naslim et al. 2011).

The middle panel of Fig. 1 shows the effective temperature against helium abundance for the stars in Table 1. The hotter stars tend to have a higher He abundance than their cooler counterparts, as expected (e.g. Edelmann et al. 2003), and as such are predominantly the p-mode pulsators. There are two stars that do not follow the general trend in the plot: the g-mode pulsator LS IV-14 116 that has both high helium abundance and a high temperature and the cool g-mode pulsator KIC 1718290. The former, as described above, has an extremely peculiar helium abundance. The latter, KIC 1718290 , was shown to be on the blue horizontal branch, rather than the EHB, by Østensen et al. (2012), which may explain its obviously different position in the effective temperature against helium abundance plot when compared to the other g-mode pulsators.

Finally, the bottom panel of Fig. 1 shows the range of pulsation period of a star against its surface gravity. Models have shown (e.g. Fontaine et al. 1998; Koen et al. 1999b) that there is an expected relationship between the pulsations in the sdBV stars and their surface gravity. The black lines plotted represent the fundamental modes of $\ell=0, n=0$ (solid line) and $\ell=2, n=0$ (dashed line) and the $\ell=1, n=1$ (dotted line) mode for p-mode pulsators. Most stars lie on the short-period side of the solid line, with some at higher surface gravities better constrained by the dashed line. It must be noted, however, that surface gravity determinations in the sdBV stars can be greatly influenced by binarity, metallicity and the pulsations, forcing models to fit higher surface gravities if the effects are not accounted for.
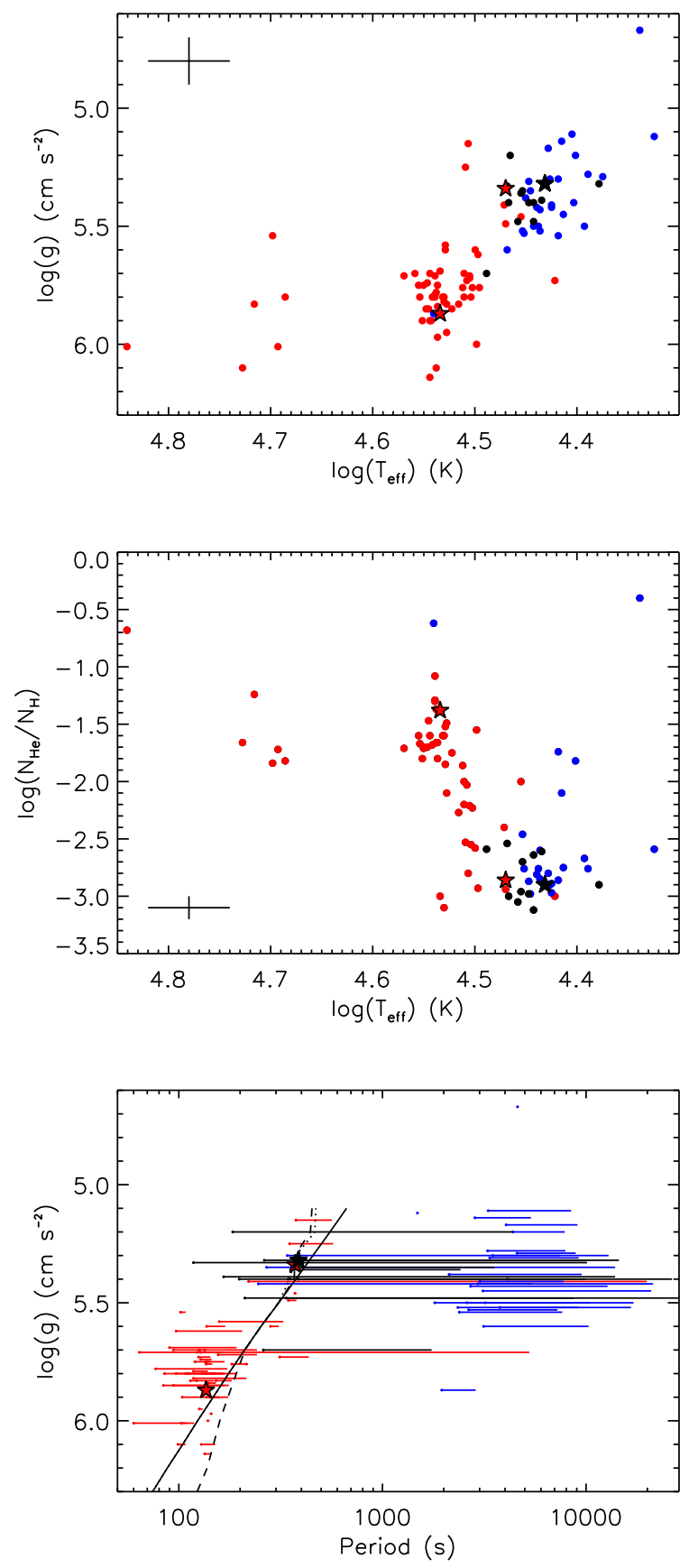

Figure 1. Top panel: the known sdV stars in the $T_{\text {eff }}-\log g$ plane for which data is available in Table 1. The red dots indicate the short-period p-mode pulsators, the blue dots are the long-period g-mode pulsators and the black dots are the hybrid pulsators. The classifications are assigned using the ground-based criterion if available, otherwise the space-based classification. The targets we report here are shown by the outlined stars. Middle panel: the $T_{\text {eff }}-\log \left(N_{\mathrm{He}} / N_{\mathrm{H}}\right)$ diagram for the stars in Table 1. In general, the hotter stars are the p-mode pulsators and are more He-rich. Bottom panel: the $P-\log g$ diagram for the same stars. The horizontal lines emanating from dots represent the range of the pulsation periods for a given star. The black lines represent the loci of models for the $\ell=0, n=0$ mode (solid line), the $\ell=2, n=0$ mode (dashed line) and the $\ell=1, n=1$ mode (dotted line) for the p-mode pulsators. See Koen et al. (1999a) for a discussion of the models. The black crosses in the top two panels indicate the typical errors. See online version for colour plots. 
Ground-based observations of the g-mode and hybrid pulsators can be challenging due to the intrinsic low amplitudes and low observational duty cycles. As such, observations by the Kepler Space Telescope have pushed the study of sdBV stars into a new age with the observations of many low-amplitude frequency-rich stars (e.g. Østensen et al. 2010a,b; Baran et al. 2011c, 2012). The precision and time-base of the observations of these stars will not be surpassed for many years.

However, with the upcoming Transiting Exoplanet Survey Satellite (TESS; Ricker et al. 2015) mission, many of the bright stars detected with ground-based observations will be revisited and be subject to $\mu$ mag precision observations for days to months at a time. TESS fields will be observed for $27 \mathrm{~d}$ at a minimum cadence of $30 \mathrm{~min}$, with 750 and 60 targets observed at 120 and $20 \mathrm{~s}$, respectively, per field. Where fields overlap, multiple 27 -d periods will combine to provide longer time-base observations, and in the best cases up to $1 \mathrm{yr}$ at the ecliptic poles. As such, the identification of further, bright, targets is key to expanding the sample size available for TESS observations, which will have a limiting magnitude of $I_{C} \lesssim 10-13$ (Ricker et al. 2015).

\subsection{The SuperWASP project}

The Super Wide Angle Search for Planets (WASP) project is a two-site wide-field survey for transiting exoplanets (Pollacco et al. 2006). The instruments are located at the Observatorio del Roque de los Muchachos on La Palma and at the Sutherland Station of the South African Astronomical Observatory. The instruments consist of eight $200 \mathrm{~mm}, \mathrm{f} / 1.8$ Canon telephoto lenses backed by Andor CCDs of $2048 \times 2048$ pixels, allowing a pixel size of about 14 arcsec. Observations are made through broad-band filters of 4000-7000 ̊. An instrument reconfiguration was conducted in 2012 on SuperWASP-S. This involved changing the $200 \mathrm{~mm}$ lenses for $85 \mathrm{~mm}$ to enable the targeting of brighter stars for planetary transits (Turner et al. 2015).

The data pass through a custom reduction pipeline correcting for primary and secondary extinctions, the colour-response of the instrument, the zero-point and atmospheric extinction. The pipeline is optimized for $\mathrm{G}$ stars. The data are also corrected for instrumental systematics using the SYSREM algorithm of Tamuz et al. (2005). The observing strategy of SuperWASP provides two consecutive $30 \mathrm{~s}$ exposures at a given pointing, before moving to the next available field; fields are typically revisited every $10 \mathrm{~min}$. Such an observing strategy allows for a nominal Nyquist frequency of $1440 \mathrm{~d}^{-1}$, but in reality due to the pseudo-random sampling there is no strict Nyquist frequency. Frequency analysis is only limited by the length of the exposure.

The SuperWASP project has been shown to have a versatile archive in which to search for a whole host of variable stars (e.g. Maxted et al. 2008; Thomas et al. 2010; Norton et al. 2011; Smalley et al. 2011, 2014, 2017; McQuillin et al. 2012; Holdsworth et al. 2014; Holdsworth 2015). In this paper, we provide an in depth analysis of three sdBV stars that have been identified to vary by Holdsworth et al. (2014) and Holdsworth (2015). These stars were not previously known to be pulsating sdB stars, and as such, provide further examples of the sdBV group of variable stars. These targets are prime candidates for follow-up observations by the TESS space mission due to their relative brightness among the sdV stars.

Throughout this paper, the reader must bear in mind that the amplitudes presented are those detected in the broad-band filter of the SuperWASP instrument. As such, they will be greatly reduced when compared to other sdV stars that are typically observed through

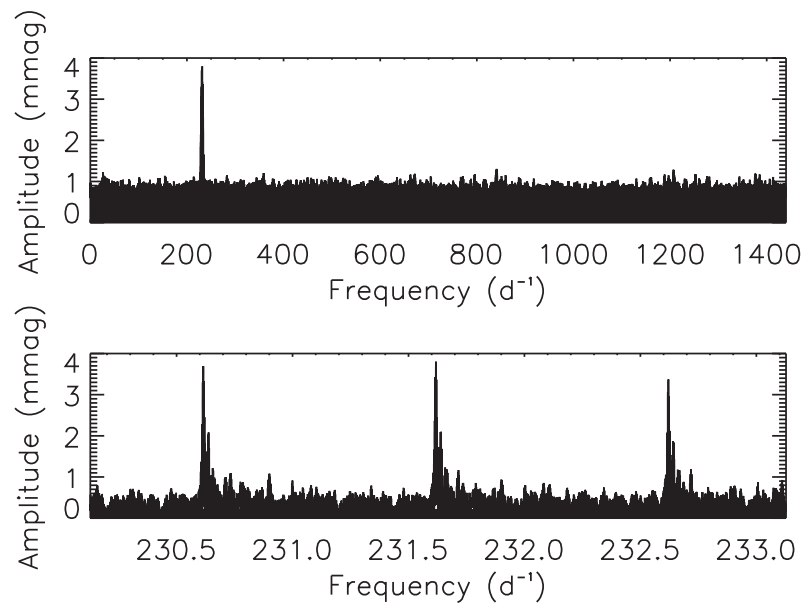

Figure 2. Top panel: amplitude spectrum of the discrete Fourier transform of all data, to the nominal Nyquist frequency, for J1938+5609 showing periodic variability at $231 \mathrm{~d}^{-1}$. Bottom panel: zoomed view of the variability and the alias structure. The second peak can be seen on the high-frequency shoulder of the main signal.

narrower band filters in the blue part of the spectrum. The pulsation amplitudes in these stars are greater in the blue part of the spectrum as a result of their high temperature and the variations in the effective temperature over the pulsation cycle.

\section{THE TARGETS}

\section{$2.1 \mathrm{~J} 1938+5609$}

$\mathrm{J} 1938+5609(\alpha: 19: 38: 32.48, \delta:+56: 09: 44.6)$ is a newly identified $\mathrm{sdB}$ star that pulsates at a frequency of $231.62 \mathrm{~d}^{-1}(P=373 \mathrm{~s})$ with an average amplitude of 4 mmag (Fig. 2). The star was observed by SuperWASP over a period of 4 yr (2007-2010), and was observed simultaneously by two different cameras in 2007 and 2008. Table 2 details the SuperWASP observations. Where there are multiple observations per season, a letter is added to the season column to aid differentiation. Peaks that had the greatest amplitude in the combined data set were taken to be the true variability of the star. The peaks that surround the peak at greatest amplitude are a result of the window pattern, which is dominated by the daily aliases that plague ground-based, single-site, time-resolved observations.

To confirm this star as an sdB star, an optical spectrum of J1938+5609 was obtained with Intermediate dispersion Spectrograph and Imaging System (ISIS) instrument mounted on the 4.2-m William Herschel Telescope (WHT) on 2015 March 12. We used the R600B grating with a 1.5 arcsec slit, attaining a resolution of $R$ $\sim 2000$. The exposure time was $1000 \mathrm{~s}$, leading to a peak $\mathrm{S} / \mathrm{N} \sim 180$. The spectrum has been reduced in the standard way, including flatfield correction, de-biasing and wavelength calibrations applied. Tools from the STARLINK project $^{1}$ were used to perform these tasks. The spectrum was intensity rectified using the UCLSYN spectral synthesis package (Smalley, Smith \& Dworetsky 2001).

The spectrum is quite featureless, with only Balmer lines and He I lines present. The hydrogen and helium lines of the extracted spectrum were fitted to a grid of synthetic spectra calculated from fully line blanketed local thermodynamic equilibrium model atmospheres assuming solar metallicity (Heber et al. 2000). The result

\footnotetext{
${ }^{1} \mathrm{http}: / /$ starlink.eao.hawaii.edu/starlink
} 
Table 2. Details of the SuperWASP observations for J1938+5609, and the results of a non-linear least-squares fitting to each of the seasons. Time (BJD) is given as BJD-2400000.0. The zero-point for the phases is taken to be the centre point of each of the data sets.

\begin{tabular}{|c|c|c|c|c|c|c|c|c|}
\hline Season & $\begin{array}{l}\text { BJD } \\
\text { start }\end{array}$ & $\begin{array}{l}\text { Length } \\
\text { (d) }\end{array}$ & $\begin{array}{l}\text { Number of } \\
\text { points }\end{array}$ & ID & $\begin{array}{l}\text { Frequency } \\
\qquad\left(\mathrm{d}^{-1}\right)\end{array}$ & $\begin{array}{l}\text { Amplitude } \\
\text { (mmag) }\end{array}$ & $\begin{array}{l}\text { Phase } \\
\text { (rad) }\end{array}$ & $\mathrm{S} / \mathrm{N}$ \\
\hline $2007 \mathrm{a}$ & 4230.5894 & 66.0356 & 2658 & $v_{1}$ & $231.6179 \pm 0.0009$ & $6.61 \pm 0.66$ & $0.106 \pm 0.113$ & 6.37 \\
\hline $2008 \mathrm{a}$ & 4631.7129 & 58.6768 & 1545 & $v_{1}$ & $231.6195 \pm 0.0019$ & $7.98 \pm 1.69$ & $0.322 \pm 0.217$ & 3.30 \\
\hline $2008 \mathrm{~b}$ & 4605.6377 & 84.9810 & 5732 & $v_{1}$ & $231.6179 \pm 0.0008$ & $5.14 \pm 0.54$ & $-1.249 \pm 0.109$ & 6.10 \\
\hline \multirow[t]{2}{*}{2009} & 4971.6289 & 127.8379 & 8489 & $v_{1}$ & $231.6189 \pm 0.0005$ & $3.89 \pm 0.39$ & $1.627 \pm 0.098$ & 6.70 \\
\hline & & & & $v_{2}$ & $231.6405 \pm 0.0007$ & $2.53 \pm 0.38$ & $-2.991 \pm 0.154$ & 4.57 \\
\hline & & & & $v_{2}$ & $231.6397 \pm 0.0008$ & $2.49 \pm 0.38$ & $-1.879 \pm 0.155$ & 5.12 \\
\hline \multirow[t]{2}{*}{ All } & 4230.5894 & 1228.9243 & 31555 & $v_{1}$ & $231.61808 \pm 0.00002$ & $3.77 \pm 0.23$ & $-0.608 \pm 0.062$ & 11.08 \\
\hline & & & & $v_{2}$ & $231.64058 \pm 0.00004$ & $1.98 \pm 0.23$ & $-0.914 \pm 0.118$ & 6.34 \\
\hline
\end{tabular}

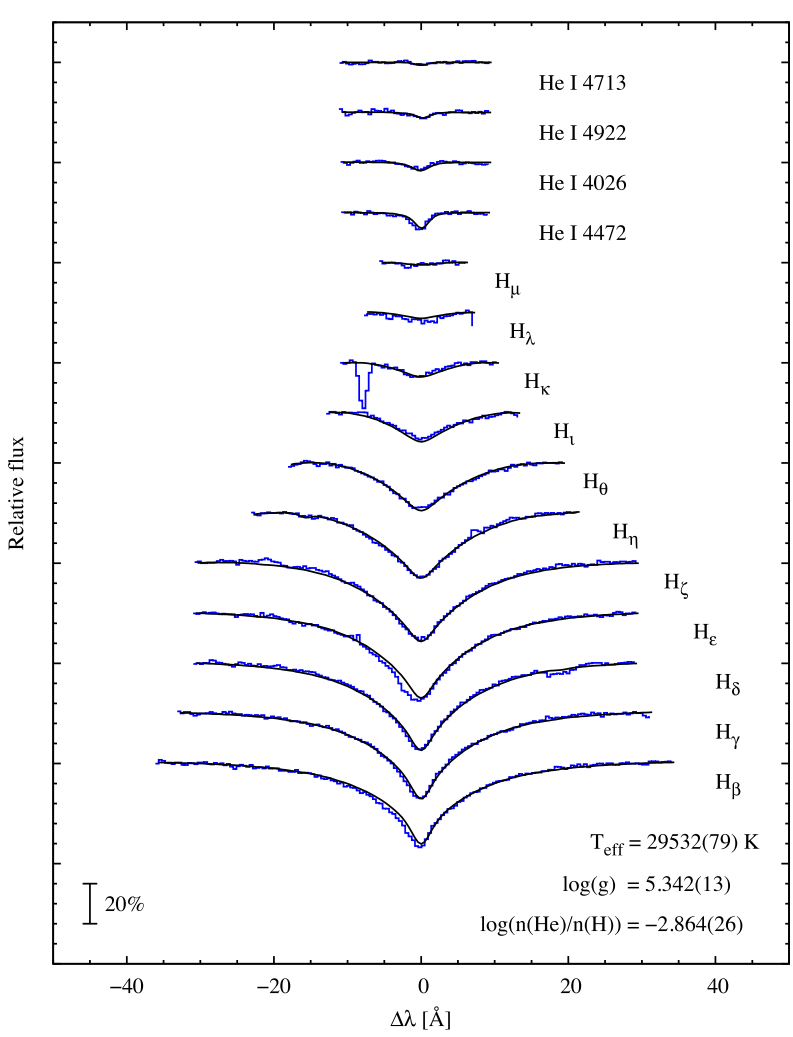

Figure 3. Fitting results of the WHT/ISIS spectrum of J1938+5609 confirming it to be an sdB star. Values in brackets of the fit parameters are the errors in the last digits, see the text for more realistic errors. The artefact in the blue wing of $\mathrm{H}_{\kappa}$ is from a bad CCD column.

is shown in Fig. 3. The artefact in the blue wing of $\mathrm{H}_{\kappa}$ is from a bad CCD column. Note that formal fitting errors stated in the figure do not account for systematic effects inherent in the models, so we generously increase the errors when stating $T_{\text {eff }}=29500 \pm 500 \mathrm{~K}$, $\log g=5.34 \pm 0.10 \mathrm{~cm} \mathrm{~s}^{-2}$ and $\log \left(N_{\mathrm{He}} / N_{\mathrm{H}}\right)=-2.86 \pm 0.10$.

To analyse the light curve, we treat each season of data separately. To remove the remaining low-frequency 'red' noise from the light curve after the data have been processed by the SuperWASP pipeline, we pre-whiten the data to $10 \mathrm{~d}^{-1}$ to an amplitude limit that is representative of the noise level at high-frequency. This frequency limit is sufficiently removed from the pulsation as to not affect the subsequent analysis. This is an iterative process where we identify, fit and remove peaks above the high-frequency noise level. We then apply linear and non-linear least-squares fitting to the light curve

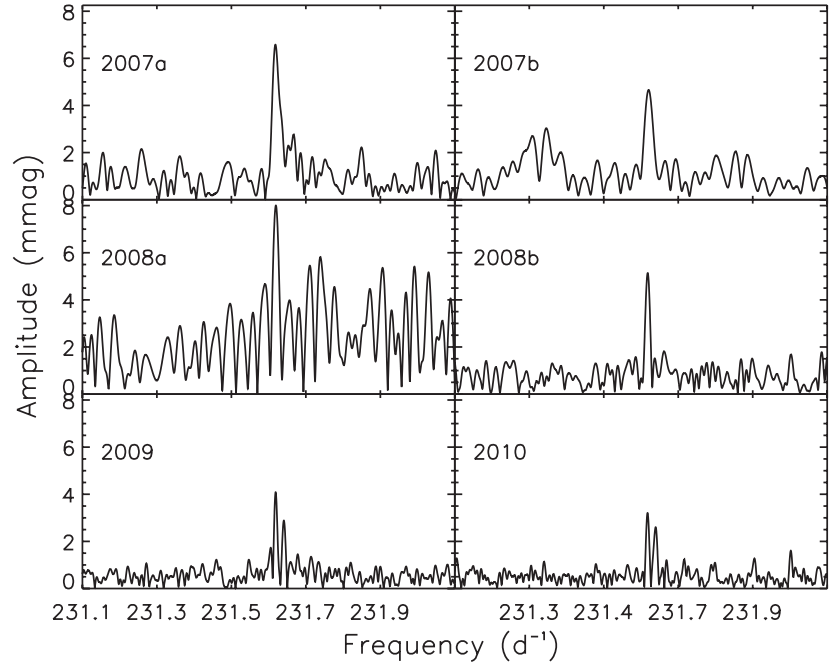

Figure 4. Amplitude spectrum of the discrete Fourier transform of the separate seasons for J1938+5609 showing the amplitude variation of the peak, and the splitting of the mode into two well-resolved frequencies.

to extract the frequency of variability. The results of the non-linear least-squares fitting are shown in Table 2.

As can be seen from the results, the amplitude of the pulsation changes over the 4-yr period. This is shown graphically in Fig. 4. The 2008a season of data is shorter in duration and low on the number of points, making the amplitude determination much more difficult, as is demonstrated by the large error in Table 2 . The other seasons show, on average, a decrease in the pulsation amplitude of the principal peak, and an eventual emergence of a second peak at $v_{2}=231.64 \mathrm{~d}^{-1}$.

The presence of the second peak in the later seasons, combined with the decrease in amplitude, suggests there may be beating between the two peaks or with further, unresolved, peaks. However, the separation of the two peaks, $\delta v=0.022 \pm 0.001 \mathrm{~d}^{-1}$, is equal to the lowest frequency resolution of the SuperWASP data (namely the $2007 \mathrm{~b}$ data). As such, the two peaks should be resolved in all the periodograms. The low quality of the WASP data does not allow us to confirm the presence of variable amplitude over the course of the observations presented here - although the results are suggestive of variability, the noise and resolution are not sufficient for its presence to be confirmed.

Very few sdBV stars show just one or two pulsation modes (see Table 1). Other than the stars identified by Brown et al. (2013) for which no accurate temperatures are published, J1938+5609 


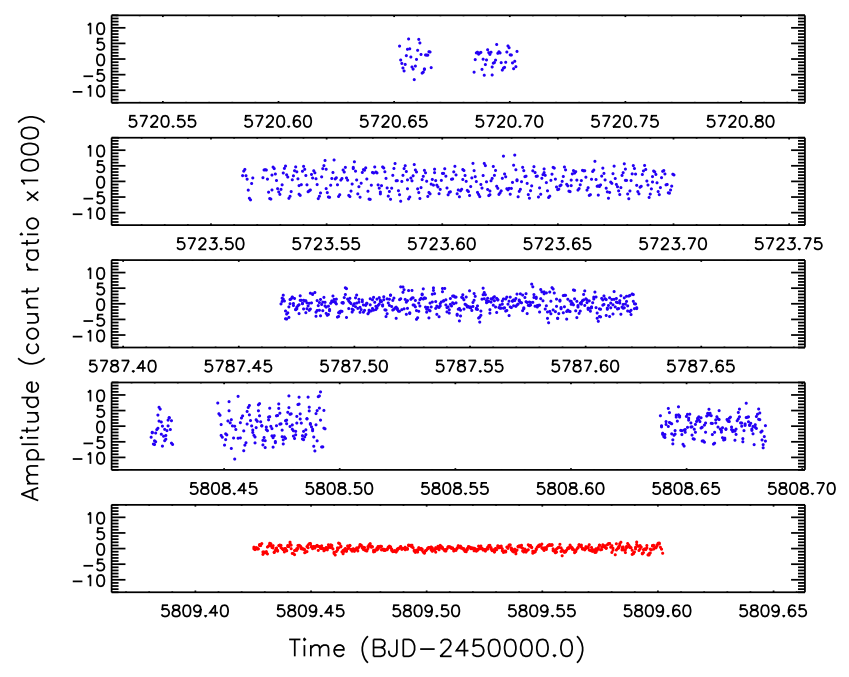

Figure 5. Mercator light curve of $\mathrm{J} 1938+5609$. Note the variable amplitude seen in the data. The observations in the top four panels, blue data points, were obtained through a BG filter, with the red data points in the bottom panel obtained through an RG filter. See the online version for colour plots.

is the second coolest single mode variable. The temperature and surface gravity of $\mathrm{J} 1938+5609$ place it among the hybrid pulsators (see Fig. 1). In terms of its stellar parameters, this target is similar to Balloon 090100001 (Baran, Pigulski \& O’Toole 2008; Telting et al. 2008; Baran et al. 2009), which shows amplitude variations in both photometric and spectroscopic observations. The variations seen in the amplitude spectrum of Balloon 090100001 are likely to be caused by energy transfer between the $\mathrm{p}$ and g modes. We therefore postulate this might be the case for $\mathrm{J} 1938+5609$, and that there may be further modes present in this star, but below our detection limit.

Independently of the SuperWASP survey, J1938+5609 was targeted as part of the survey for bright pulsators in the GALEX sample, which also yielded J20133+0928 (Østensen et al. 2011b), J08069+1527 (Baran et al. 2011b) and J06398+5156 (Vučković et al. 2012). All observations were obtained with the Merope II frame-transfer imager on the 1.2-m Mercator telescope (Østensen 2010). The data were processed by standard overscan bias-level subtraction and flat-fielding, and the light curves extracted by aperture photometry, using the Real Time Photometry (RPT) program (Østensen et al. 2001a). Observations were made through two different filters: the $R$ and $B$ filters of the Geneva system denoted RG and BG, respectively. The targets were first observed on the night of 2011 June 7 during two short runs. Those discovery runs were immediately followed up with a long run on 2011 June 10. Another fairly long run was obtained on the night of 2011 August 13 during the same campaign as for FBS 0117+396 (Østensen et al. 2013), while waiting for that target to become high enough to observe. Finally, it was observed on the nights of 2011 September 3 and 4, where the observations on the first of those nights suffered from intermittent clouds.

The extracted light curves are shown in Fig. 5. The star shows amplitude variability over the period of the observations. However, the data are not of sufficient length to resolve more than one mode, as seen in SuperWASP. The manner in which the data are extracted with the RPT programme provides a magnitude in counts relative to the comparison stars. As such, the extraction of amplitude from the data for comparison to the SuperWASP white light data is not possible. The data do, however, demonstrate the amplitude suppres-
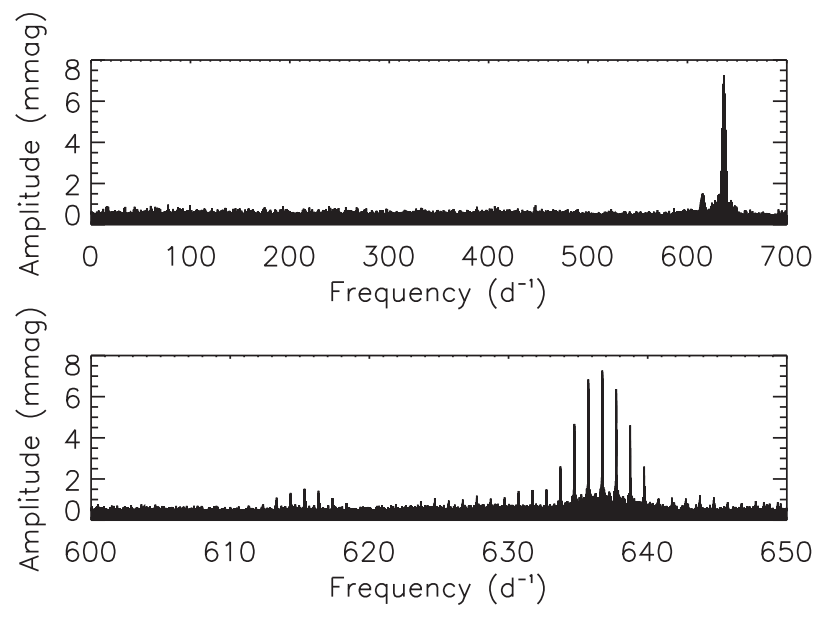

Figure 6. Top panel: full amplitude spectrum of the discrete Fourier transform of the SuperWASP data of J0902-0720 showing two periodic variations. Bottom panel: zoomed view of the variations seen in the top panel.

sion as a function of filter response, and thus the need for blue-band observations of these stars to maximize frequency detection.

\section{$2.2 \mathrm{~J} 0902-0720$}

J0902-0720 ( $\alpha$ : 09:02:04.52, $\delta:-07: 20: 47.58$; TYC 4890-19-1) is a newly identified $\mathrm{sdB}$ star, detected through the light variations in SuperWASP data. The data show variability at $636.74 \mathrm{~d}^{-1}$ $(P=136 \mathrm{~s})$ with an average amplitude of $7.27 \mathrm{mmag}$, and a second peak at $615.34 \mathrm{~d}^{-1}(P=140 \mathrm{~s})$ with an average amplitude of 1.53 mmag (Fig. 6).

A low-resolution spectrum $(R \sim 1360)$ of J0902-0720 was obtained with the Andalucia Faint Object Spectrograph and Camera, ALFOSC, instrument mounted on the 2.56-m Nordic Optical Telescope (NOT), on 2013 February 04. We used grism \#16 and a 1.0 arcsec slit. The exposure time was $300 \mathrm{~s}$, leading to a peak $\mathrm{S} / \mathrm{N} \sim 220$ in the extracted spectrum. Standard reduction steps within IRAF include bias subtraction, removal of pixel-to-pixel sensitivity variations, optimal spectral extraction and wavelength calibration based on arc-lamp spectra. There are many He lines in the spectrum, with very few other features (excluding the $\mathrm{H}$ lines). The results of the spectral fitting are shown in Fig. 7, where the errors shown are the formal fitting errors. When taking into account systematic effects, we adopt $T_{\text {eff }}=34200 \pm 500 \mathrm{~K}, \log g=5.87 \pm 0.10 \mathrm{~cm} \mathrm{~s}^{-2}$ and $\log \left(N_{\mathrm{He}} / N_{\mathrm{H}}\right)=-1.38 \pm 0.1$, confirming this star as a pulsating sdB star. These parameters place the star amongst the p-mode pulsators.

To analyse the light curve of J0902-0720, we pre-whitened each season of data to $10 \mathrm{~d}^{-1}$ to an amplitude representative of the high-frequency noise, following the procedure described earlier. We then conducted linear and non-linear least-squares fitting of the pulsations. The results of the non-linear least-squares fitting are shown in Table 3. As before, seasons with multiple data are differentiated by a letter, and peaks are identified from the aliases in the amplitude spectrum of the combined data.

We detect the principal peak in all available seasons of SuperWASP data. The second peak, $v_{2}$ at $615.34 \mathrm{~d}^{-1}$, is detected in all bar one season. That season, 2011a, has the shortest length and a noise level of about 2.4 mmag at the frequency of the undetected peak. A third peak, $v_{3}$ at $615.44 \mathrm{~d}^{-1}$, is detected in two of the seasons. This has a similar amplitude to $v_{2}$ and as such should be detected 


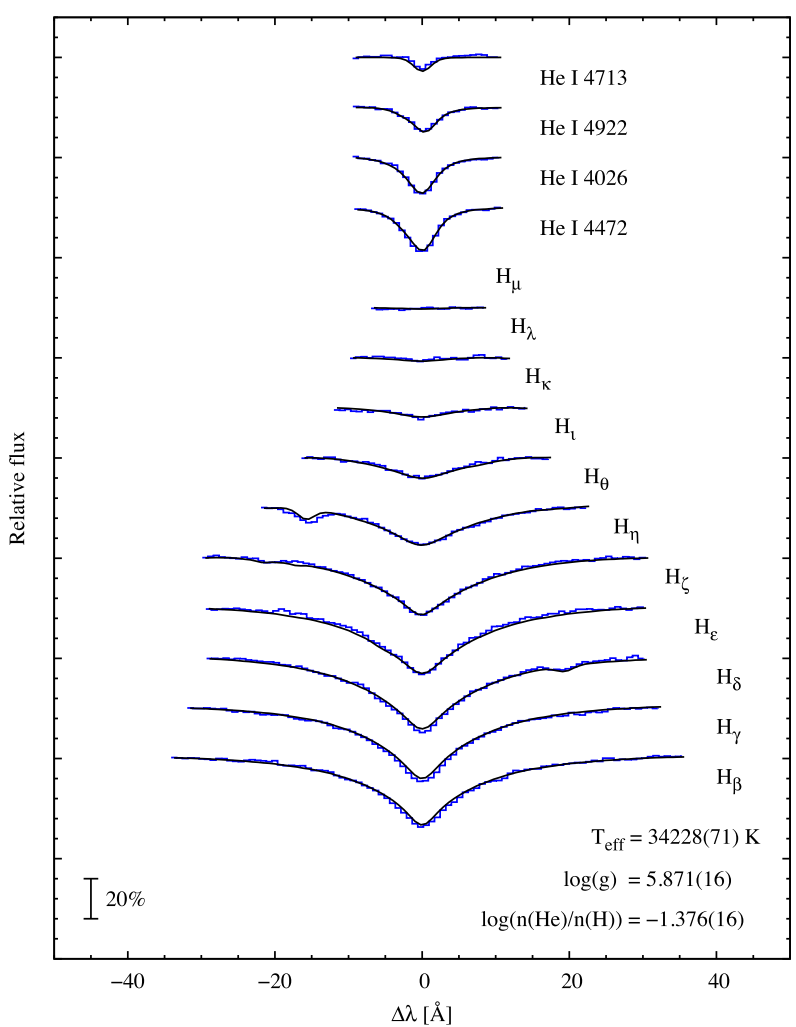

Figure 7. The results of spectral fitting of the NOT/ALFOSC spectrum of J0902-0720. Values in brackets of the fit parameters are the errors in the last digits. See the text for more realistic errors.

in most of the data sets. Further data are required to confirm $v_{3}$ as a real signal.

Assuming that the peaks seen in the periodogram of J0902-0720 at $136 \mathrm{~s}$ are typical of those found in other sdBV stars, we conclude that the light variations are due to p-mode pulsations driven by the iron opacity bump (Østensen 2010).

It must be noted here that the amplitudes of the pulsations in J0902-0720 will be reduced as a result of the relatively long exposure time of the SuperWASP observations, when compared to the pulsation period. Taking this into account, the amplitude is reduced by 8 per cent in the SuperWASP passband. This is calculated using the relation

$\frac{A}{A_{0}}=\operatorname{sinc} \frac{\pi T_{\text {exp }}}{P_{\text {puls }}}$,

where $A$ is the measured amplitude, $A_{0}$ is the intrinsic amplitude (in the observed passband), $T_{\exp }$ is the exposure time which in the case of SuperWASP is $30 \mathrm{~s}$ and $P_{\text {puls }}$ is the pulsation period.

The spectroscopic parameters derived for J0902-0720 place it among the p-mode pulsators (Fig. 1). The temperature and surface gravity suggest no g modes would likely be present in the star. Further to this, the He abundance we derive for J0902-0720 is high. When compared to the other sdBV stars (see middle panel of Fig. 1), there are few p-mode pulsators that show a higher $\mathrm{He}$ abundance. The position that J0902-0720 occupies in that diagram is similar to $2 \mathrm{M} 0415+0154$, HE $1450-0957$ and $\mathrm{J} 23341+4622$ all of which show just two or three pulsation modes. However, we cannot rule out the presence of further modes in J0902-0720 from our broad-band photometry. This bright $(V=12.4)$ target is ideal for dedicated follow-up observations.

\subsection{J2344-3427}

J2344-3427 ( $\alpha: 23: 44: 22.01, \delta:-34: 27: 00.40 ;$ HE 2341-3443) was included in the survey of Østensen et al. (2010c), but no significant pulsations were detected in that short run. With only $\sim 800$ data points, the detection limit was just $\sim 3 \mathrm{mmag}$, so their null detection is not in conflict with the SuperWASP detection presented here.

The SuperWASP observations of J2344-3427 cover five seasons, with a total of 43572 data points. To analyse the high-frequency

Table 3. Details of the SuperWASP observations for J0902-0720, and the results of a non-linear least-squares fitting to each of the seasons. Time (BJD) is given as BJD-2400000.0. The zero-point for the phases is taken to be the centre point of each of the data sets.

\begin{tabular}{|c|c|c|c|c|c|c|c|c|}
\hline Season & $\begin{array}{l}\text { BJD } \\
\text { start }\end{array}$ & $\begin{array}{l}\text { Length } \\
\text { (d) }\end{array}$ & $\begin{array}{c}\text { Number of } \\
\text { points }\end{array}$ & ID & $\begin{array}{l}\text { Frequency } \\
\qquad\left(d^{-1}\right)\end{array}$ & $\begin{array}{l}\text { Amplitude } \\
\text { (mmag) }\end{array}$ & $\begin{array}{l}\text { Phase } \\
\text { (rad) }\end{array}$ & $\mathrm{S} / \mathrm{N}$ \\
\hline \multirow[t]{2}{*}{$2009 a$} & 4846.3565 & 97.0020 & 4652 & $v_{1}$ & $636.7340 \pm 0.0002$ & $12.14 \pm 0.50$ & $-1.184 \pm 0.041$ & 9.15 \\
\hline & & & & $v_{3}$ & $615.4361 \pm 0.0012$ & $2.19 \pm 0.50$ & $-1.107 \pm 0.233$ & 3.84 \\
\hline \multirow[t]{2}{*}{$2009 b$} & 5167.6401 & 118.8760 & 2908 & $v_{1}$ & $636.7356 \pm 0.0002$ & $9.59 \pm 0.47$ & $2.092 \pm 0.051$ & 7.75 \\
\hline & & & & $v_{2}$ & $615.3393 \pm 0.0011$ & $1.91 \pm 0.48$ & $-2.868 \pm 0.256$ & 3.22 \\
\hline \multirow[t]{3}{*}{ 2010a } & 5212.4082 & 95.9702 & 4119 & $v_{1}$ & $636.7358 \pm 0.0003$ & $11.77 \pm 0.52$ & $1.845 \pm 0.044$ & 8.95 \\
\hline & & & & $v_{2}$ & $615.3403 \pm 0.0015$ & $2.11 \pm 0.54$ & $-2.229 \pm 0.246$ & 3.93 \\
\hline & & & & $v_{3}$ & $615.4376 \pm 0.0012$ & $2.62 \pm 0.54$ & $-1.404 \pm 0.203$ & 3.66 \\
\hline \multirow[t]{2}{*}{ 2010b } & 5532.7612 & 116.7583 & 3759 & $v_{1}$ & $636.7371 \pm 0.0002$ & $9.60 \pm 0.35$ & $0.058 \pm 0.038$ & 8.34 \\
\hline & & & & $v_{2}$ & $615.3411 \pm 0.0007$ & $2.44 \pm 0.35$ & $-2.013 \pm 0.148$ & 5.06 \\
\hline $2010 \mathrm{c}$ & 5543.5894 & 105.9302 & 3735 & $v_{1}$ & $636.7375 \pm 0.0002$ & $10.53 \pm 0.37$ & $2.208 \pm 0.035$ & 8.72 \\
\hline \multirow{2}{*}{ All } & & & & $v_{2}$ & $615.339722 \pm 0.00006$ & $1.59 \pm 0.18$ & $-3.023 \pm 0.116$ & 6.26 \\
\hline & & & & $v_{3}$ & $615.435681 \pm 0.00009$ & $0.94 \pm 0.18$ & $-0.149 \pm 0.197$ & 3.71 \\
\hline
\end{tabular}



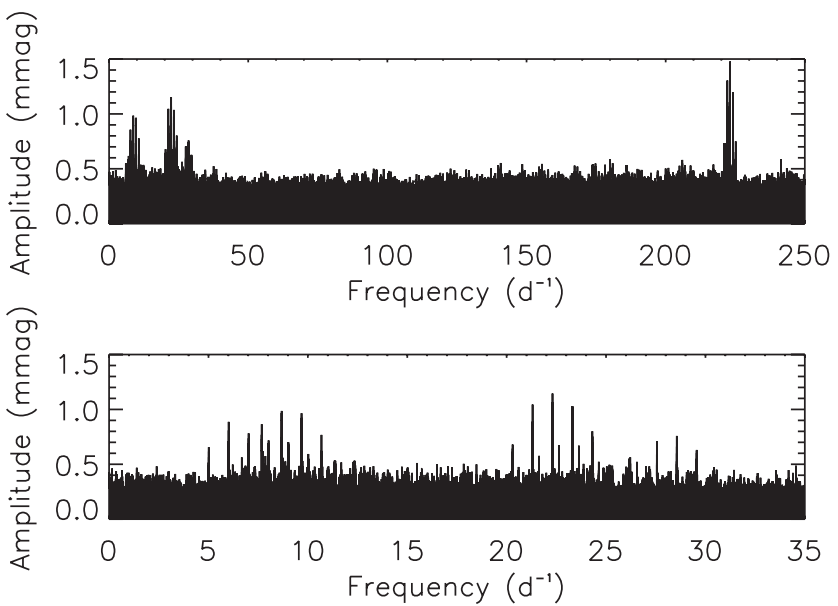

Figure 8. Top panel: full amplitude spectrum of the discrete Fourier transform of the SuperWASP light curve of J2344-3427 using the first three seasons of data. Bottom panel: the low-frequency range shown in more detail due to the presence of multiple frequencies.

variability in this star, we pre-whiten each individual light curve to a frequency of $10 \mathrm{~d}^{-1}$ and an amplitude equalling the approximate noise level of the high-frequency range, as performed for the previous targets. When analysing the peaks in the low-frequency regime, we do not pre-whiten the data - as the peaks are close to the red noise peaks in frequency space, altering the light curve at low frequency to remove noise may affect the intrinsic variability signal.

The high-frequency variation, at $223.16 \mathrm{~d}^{-1},(P=387 \mathrm{~s})$ is detected in all seasons of the data with an average amplitude of $1.5 \mathrm{mmag}$ (Fig. 8 top panel). As well as the high-frequency variability, there are further frequencies at $8.68,22.31$ and $28.56 \mathrm{~d}^{-1}$ $(P=9954,3873$ and $3025 \mathrm{~s})$ with amplitudes of $0.99,1.17$ and 0.76 mmag, respectively (Fig. 8 bottom panel). These lowfrequency variations are only detected (with significance) in the first three seasons of data. The lack of detections in the 2012 and 2013 seasons is most likely a result of the change to $85 \mathrm{~mm}$ lenses, which results in a lower photometric precision per observation, and hence a higher noise level in the periodogram. The 2006, 2007 and 2011 seasons achieve a noise level of $\sim 0.7 \mathrm{mmag}$ in the low-frequency range, whereas the 2012 and 2013 data reach just 1.4 mmag, greater than the detected pulsation amplitudes. A full frequency analysis, as well as a log of the SuperWASP observations, is shown in Table 4.

These low-frequency pulsations may originate from a mainsequence companion, as most sdB stars are found in binary systems, or they may be $\mathrm{g}$ modes in the same star, as is observed with the DW Lyn sdBV stars (Schuh et al. 2006). However, we are able to rule-out some binary scenarios: the lack of a detected orbital period, or its harmonic, allows us to exclude a short-period binary (such as HW Vir); a $\gamma$ Dor or $\delta$ Sct star would be detected in the spectrum of J2344-3427, which was shown not to be the case by Geier \& Heber (2012), thus we can exclude these A/F star pulsators from contaminating the light curve; and the lack of any IR excess in 2MASS photometry allows us to exclude sufficiently luminous stars that could have pulsations in our light curve. We are confident, therefore, that $\mathbf{J} 2344-3427$ is a single star that shows hybrid pulsations.

There are conflicting temperatures for J2344-3427 presented in the literature. McDonald, Zijlstra \& Boyer (2012) measured the effective temperature of J2344-3427 to be $37448 \pm 1648 \mathrm{~K}$. This value is derived through SED fitting and does not take into account interstellar reddening. Furthermore, they use blackbody fits that are not appropriate for hot stars. A more accurate result is presented by Geier et al. (2013) who used their FEROS (The Fiber-fed Extended Range Optical Spectrograph) spectrum to derive a $T_{\text {eff }}$ of $27000 \pm 500 \mathrm{~K}$. Further to this, Heber et al. (1984) found a value of $28800 \pm 1500 \mathrm{~K}$ and a $\log g$ of $5.4 \pm 0.2 \mathrm{~cm} \mathrm{~s}^{-2}$, and Németh et al. (2012) found a $T_{\text {eff }}$ of $28390 \pm 265 \mathrm{~K}$. These values from spectral fitting provide a much more reliable temperature estimate than that of McDonald et al. (2012). Therefore we adopt $T_{\text {eff }}=28000 \pm 250 \mathrm{~K}$ for this star by taking the weighted mean and its error from the spectroscopically derived temperatures. Such a temperature places the star in the hybrid star temperature range. Given that only one measurement of $\log g$ was found, we adopt that value.

Previous to Kepler, these hybrid pulsators were seen to have amplitudes much larger than their non-hybrid counterparts. However, Reed et al. (2010b) have found pulsations in Kepler targets that would have been below ground-based detection limits. Kepler observations have also shown g modes to be of higher amplitude than the $\mathrm{p}$ modes in the same star, unlike previously observed hybrid sdBV stars, as well as J2344-3427 here.

The presence of both $\mathrm{p}$ and $\mathrm{g}$ modes in a single star is key to understanding the structure of the star as the different excitation mechanisms probe different depths. Further observations of J2344-3427 are required to confirm the presence of the $\mathrm{g}$ modes, and provide a full frequency solution for asteroseismological modelling.

\section{SUMMARY AND CONCLUSIONS}

As there are relatively few variable hot subdwarf stars known (Table 1), the identification of further class members is key to understanding these stars. A previous attempt to identify new sdBV stars in the SuperWASP archive only resulted in the confirmation of previously known variables (Maxted et al. 2008). However, in this work, we have identified and analysed three new sdBV stars found in the SuperWASP archive. Two of these stars, J0902-0720 and $\mathrm{J} 1938+5609$, are of the short-period p-mode type, while the third, J2344-3427, is a hybrid pulsator.

Amongst the short-period stars, J1938+5609 is at the lower temperature and surface gravity end of the distribution (Fig. 1), and as such is mixed with the long-period pulsators in the $T_{\text {eff }}-\log g$ plane. The reason for this is unclear. However, the noise limits of the SuperWASP data do not allow us to rule-out the presence of low-amplitude g modes that would make this star a hybrid pulsator. It would then fall in the expected region in the $T_{\text {eff }}-\log g$ plane. The position of J0902-0720 in the blue part of that plane suggests that $\mathrm{g}$ modes are unlikely to be found in this star.

J2344-3427 has been identified as a hybrid pulsator, and is found amongst the other hybrid stars in the $T_{\text {eff }}-\log g$ plane. Given the location of $\mathrm{J} 1938+5609$ is similar to $\mathrm{J} 2344-3427$ in the $T_{\text {eff }}-\log g$, we postulate that $\mathrm{J} 1938+5609$ star is also a hybrid pulsator. The amplitude of the g-mode pulsations in J2344-3427 are of the order of the noise level of J1938+5609 (in the same frequency range), further suggesting that J1938+5609 may be a hybrid pulsator (as defined in the limit of ground-based observations) with the signal lost in the noise. Additional observations, in the appropriate passband, are required to fully characterize all these targets. These observations may lead to the identification of further, low-amplitude, pulsations not identified in our broad-band observations.

The stars presented in this work demonstrate the ability of survey data to identify unknown sdBV stars, and thus expand the 
Table 4. Details of the SuperWASP observations for J2344-3427, and the results of a non-linear least-squares fitting to each of the seasons. Time (BJD) is given as BJD-2400000.0. The zero-point for the phases is taken to be the centre point of each of the data sets.

\begin{tabular}{|c|c|c|c|c|c|c|c|c|}
\hline Season & $\begin{array}{l}\text { BJD } \\
\text { start }\end{array}$ & $\begin{array}{l}\text { Length } \\
\text { (d) }\end{array}$ & $\begin{array}{c}\text { Number of } \\
\text { points }\end{array}$ & ID & $\begin{array}{l}\text { Frequency } \\
\qquad\left(d^{-1}\right)\end{array}$ & $\begin{array}{c}\text { Amplitude } \\
\text { (mmag) }\end{array}$ & $\begin{array}{l}\text { Phase } \\
\text { (rad) }\end{array}$ & $\mathrm{S} / \mathrm{N}$ \\
\hline \multirow[t]{3}{*}{2006} & 3870.6143 & 183.8618 & 4574 & $v_{1}$ & $223.1601 \pm 0.0003$ & $1.46 \pm 0.16$ & $1.240 \pm 0.096$ & 6.78 \\
\hline & & & & $v_{3}$ & $22.3065 \pm 0.0004$ & $1.37 \pm 0.16$ & $-2.851 \pm 0.116$ & 5.17 \\
\hline & & & & $v_{4}$ & $28.5596 \pm 0.0006$ & $0.92 \pm 0.16$ & $2.432 \pm 0.176$ & 4.48 \\
\hline \multirow[t]{2}{*}{2007} & 4268.5259 & 164.9025 & 4558 & $v_{1}$ & $223.1599 \pm 0.0004$ & $1.40 \pm 0.23$ & $-1.695 \pm 0.122$ & 4.98 \\
\hline & & & & $v_{2}$ & $8.6818 \pm 0.0003$ & $2.32 \pm 0.23$ & $-1.892 \pm 0.101$ & 4.33 \\
\hline \multirow[t]{4}{*}{2011} & 5731.5244 & 153.9233 & 3860 & $v_{1}$ & $223.1602 \pm 0.0004$ & $1.78 \pm 0.21$ & $-1.056 \pm 0.108$ & 5.96 \\
\hline & & & & $v_{2}$ & $8.3442 \pm 0.0009$ & $0.91 \pm 0.21$ & $2.196 \pm 0.230$ & 3.08 \\
\hline & & & & $v_{3}$ & $22.3074 \pm 0.0005$ & $1.50 \pm 0.21$ & $-0.279 \pm 0.138$ & 5.15 \\
\hline & & & & $v_{4}$ & $28.5623 \pm 0.0010$ & $0.77 \pm 0.21$ & $-0.502 \pm 0.269$ & 2.92 \\
\hline 2012 & 6111.5190 & 148.8671 & 17174 & $v_{1}$ & $223.1622 \pm 0.0011$ & $1.44 \pm 0.39$ & $2.091 \pm 0.291$ & 2.87 \\
\hline
\end{tabular}

number that are available for in-depth analysis via targeted photometry and/or spectroscopy. Further to this, as ground-based surveys often target bright stars, the stars presented here are ideal candidates for follow-up observations by the TESS mission.

\section{ACKNOWLEDGEMENTS}

This paper is based on observations made with the Mercator-, Nordic Optical- and William Herschel Telescopes (WHT) all operated on the island of La Palma, Spain. DLH thanks the STFC for financial support via grant ST/M000877/1. The SuperWASP project is funded and operated by Queen's University Belfast, the Universities of Keele, St. Andrews and Leicester, the Open University, the Isaac Newton Group, the Instituto de Astrofísica de Canarias, the South African Astronomical Observatory and by the STFC. This work was based on service observations made with the WHT operated on the island of La Palma by the Isaac Newton Group in the Spanish Observatorio del Roque de los Muchachos of the Instituto de Astrofísica de Canarias, observations made with the Mercator Telescope, operated on the island of La Palma by the Flemmish Community, at the Spanish Observatorio del Roque de los Muchachos of the Instituto de Astrofísica de Canarias and observations made with the Nordic Optical Telescope, operated by the Nordic Optical Telescope Scientific Association at the Observatorio del Roque de los Muchachos, La Palma, Spain, of the Instituto de Astrofisica de Canarias, with ALFOSC, which is provided by the Instituto de Astrofisica de Andalucia (IAA) under a joint agreement with the University of Copenhagen and NOTSA. We thank the referee, Simon Jeffery, for useful comments and suggestions that have improved the manuscript.

\section{REFERENCES}

Aerts C., Jeffery C. S., Fontaine G., Dhillon V. S., Marsh T. R., Groot P., 2006, MNRAS, 367, 1317

Ahmad A., Jeffery C. S., 2003, A\&A, 402, 335

Ahmad A., Jeffery C. S., 2005, A\&A, 437, L51

Baran A. S., 2012, Acta Astron., 62, 179

Baran A. S., Østensen R. H., 2013, Acta Astron., 63, 79

Baran A. S., Winans A., 2012, Acta Astron., 62, 343
Baran A., Pigulski A., Kozieł D., Ogłoza W., Silvotti R., Zoła S., 2005, MNRAS, 360, 737

Baran A., Pigulski A., O’Toole S. J., 2008, MNRAS, 385, 255

Baran A. et al., 2009, MNRAS, 392, 1092

Baran A. S., Gilker J. T., Fox-Machado L., Reed M. D., Kawaler S. D., 2011a, MNRAS, 411, 776

Baran A. S., Gilker J. T., Reed M. D., Østensen R. H., Telting J. H., Smolders K., Hicks L., Oreiro R., 2011b, MNRAS, 413, 2838

Baran A. S. et al., 2011c, MNRAS, 414, 2871

Baran A. S. et al., 2012, MNRAS, 424, 2686

Baran A. S., Telting J. H., Németh P., Bachulski S., Krzesiński J., 2015, A\&A, 573, A52

Barlow B. N. et al., 2010, MNRAS, 403, 324

Barlow B. N., Dunlap B. H., Gray A. E., Clemens J. C., 2009, AJ, 138, 686

Billères M., Fontaine G., Brassard P., Charpinet S., Liebert J., Saffer R. A., Vauclair G., 1997, ApJ, 487, L81

Billères M., Fontaine G., Brassard P., Charpinet S., Liebert J., Saffer R. A., Bergeron P., Vauclair G., 1998, ApJ, 494, L75

Billéres M., Fontaine G., Brassard P., Charpinet S., Liebert J., Saffer R. A., 2000, ApJ, 530, 441

Blanchette J.-P., Chayer P., Wesemael F., Fontaine G., Fontaine M., Dupuis J., Kruk J. W., Green E. M., 2008, ApJ, 678, 1329

Bonanno A., Catalano S., Frasca A., Mignemi G., Paternò L., 2003, A\&A, 398,283

Brassard P., Fontaine G., Billères M., Charpinet S., Liebert J., Saffer R. A., 2001, ApJ, 563, 1013

Brown T. M., Sweigart A. V., Lanz T., Landsman W. B., Hubeny I., 2001, ApJ, 562, 368

Brown T. M., Landsman W. B., Randall S. K., Sweigart A. V., Lanz T., 2013, ApJ, 777, L22

Charpinet S., Fontaine G., Brassard P., Chayer P., Rogers F. J., Iglesias C. A., Dorman B., 1997, ApJ, 483, L123

Charpinet S., Fontaine G., Brassard P., Green E. M., Chayer P., 2005a, A\&A, 437, 575

Charpinet S., Fontaine G., Brassard P., Billères M., Green E. M., Chayer P., 2005b, A\&A, 443, 251

Charpinet S. et al., 2006, A\&A, 459, 565

Charpinet S. et al., 2010, A\&A, 516, L6

Charpinet S. et al., 2011, A\&A, 530, A3

Chayer P., Fontaine M., Fontaine G., Wesemael F., Dupuis J., 2006, Balt. Astron., 15, 131

Dreizler S., Schuh S. L., Deetjen J. L., Edelmann H., Heber U., 2002, A\&A, 386,249 
Drilling J. S., Jeffery C. S., Heber U., Moehler S., Napiwotzki R., 2013, A\&A, 551, A31

Edelmann H., 2003, PhD thesis, Friedrich-Alexander Univ. ErlangenNürnberg

Edelmann H., Heber U., Hagen H.-J., Lemke M., Dreizler S., Napiwotzki R., Engels D., 2003, A\&A, 400, 939

Fontaine G., Charpinet S., Brassard P., Chayer P., Rogers F. J., Iglesias C. A., Dorman B., 1998, in Deubner F.-L., Christensen-Dalsgaard J., Kurtz D., eds, Proc. IAU Symp. 185, New Eyes to See Inside the Sun and Stars. Kluwer, Dordrecht, p. 367

Fontaine G., Brassard P., Charpinet S., Green E. M., Chayer P., Billères M., Randall S. K., 2003, ApJ, 597, 518

Fontaine G., Brassard P., Green E. M., Chayer P., Charpinet S., Andersen M., Portouw J., 2008, A\&A, 486, L39

For B.-Q. et al., 2006, ApJ, 642, 1117

Foster H. M., Reed M. D., Telting J. H., Østensen R. H., Baran A. S., 2015, ApJ, 805, 94

Geier S., Heber U., Przybilla N., Kudritzki R.-P., 2006, Balt. Astron., 15, 243

Geier S., Heber U., Podsiadlowski P., Edelmann H., Napiwotzki R., Kupfer T., Müller S., 2010, A\&A, 519, A25

Geier S. et al., 2011, A\&A, 530, A28

Geier S., Heber U., 2012, A\&A, 543, A149

Geier S. et al., 2012, in Kilkenny D., Jeffery C. S., Koen C., eds, ASP Conf. Ser. Vol. 452, Fifth Meeting on Hot Subdwarf Stars and Related Objects. Astron. Soc. Pac., San Francisco, p. 129

Geier S., Heber U., Edelmann H., Morales-Rueda L., Kilkenny D., O'Donoghue D., Marsh T. R., Copperwheat C., 2013, A\&A, 557, A122

Green E. M. et al., 2003, ApJ, 583, L31

Green E. M. et al., 2011, ApJ, 734, 59

Han Z., Podsiadlowski P., Maxted P. F. L., Marsh T. R., Ivanova N., 2002, MNRAS, 336, 449

Han Z., Podsiadlowski P., Maxted P. F. L., Marsh T. R., 2003, MNRAS, 341, 669

Heber U., 1986, A\&A, 155, 33

Heber U., 2009, ARA\&A, 47, 211

Heber U., 2016, PASP, 128, 082001

Heber U., Hunger K., Jonas G., Kudritzki R. P., 1984, A\&A, 130, 119

Heber U., Reid I. N., Werner K., 1999, A\&A, 348, L25

Heber U., Reid I. N., Werner K., 2000, A\&A, 363, 198

Holdsworth D. L., 2015, PhD thesis, Keele Univ.

Holdsworth D. L. et al., 2014, MNRAS, 439, 2078

Jeffery C. S., Ramsay G., 2014, MNRAS, 442, L61

Jeffery C. S., Saio H., 2006, MNRAS, 372, L48

Jeffery C. S., Dhillon V. S., Marsh T. R., Ramachandran B., 2004, MNRAS, 352,699

Jeffery C. S., Aerts C., Dhillon V. S., Marsh T. R., Gänsicke B. T., 2005, MNRAS, 362, 66

Kawaler S. D. et al., 2010, MNRAS, 409, 1487

Ketzer L., Baran A., Reed M., Telting J. H., Nemeth P., 2016, American Astronomical Society Meeting Abstracts, \#228

Kilkenny D., Koen C., 2016, MNRAS, 457, 723

Kilkenny D., Koen C., O’Donoghue D., Stobie R. S., 1997, MNRAS, 285, 640

Kilkenny D., O’Donoghue D., Koen C., Gray A. E., van Wyk F., 1998, MNRAS, 296, 329

Kilkenny D. et al., 1999, MNRAS, 303, 525

Kilkenny D. et al., 2002, MNRAS, 331, 399

Kilkenny D. et al., 2003, MNRAS, 345, 834

Kilkenny D., Kotze J. P., Jurua E., Brownstone M., Babiker H. A., 2006a, Balt. Astron., 15, 255

Kilkenny D., O’Donoghue D., Reed M. D., Hambly N., McGillivray H., 2006b, Balt. Astron., 15, 317

Kilkenny D., Stobie R. S., O’Donoghue D., Koen C., Hambly N., MacGillivray H., Gray A. E., 2006c, MNRAS, 367, 1603

Kilkenny D., Copley C., Zietsman E., Worters H., 2007, MNRAS, 375, 1325

Kilkenny D., O’Donoghue D., Crause L., Engelbrecht C., Hambly N., MacGillivray H., 2009, MNRAS, 396, 548
Kilkenny D., Koen C., Worters H., 2010, MNRAS, 404, 376

Koen C., 2007, MNRAS, 377, 1275

Koen C., 2009, MNRAS, 395, 979

Koen C., 2011, MNRAS, 415, 3042

Koen C., Green E. M., 2007, MNRAS, 377, 1605

Koen C., Green E. M., 2010, MNRAS, 406, 2701

Koen C., O'Donoghue D., Kilkenny D., Gray A. E., Marang F., van Wyk F., 1998a, MNRAS, 296, 317

Koen C., O’Donoghue D., Pollacco D. L., Nitta A., 1998b, MNRAS, 300, 1105

Koen C., O’Donoghue D., Pollacco D. L., Charpinet S., 1999a, MNRAS, 305, 28

Koen C., O’Donoghue D., Kilkenny D., Stobie R. S., Saffer R. A., 1999b, MNRAS, 306, 213

Koen C., O’Donoghue D., Kilkenny D., Pollacco D. L., 2004, New Astron., 9,565

Koen C., Kilkenny D., Pretorius M. L., Frew D. J., 2010, MNRAS, 401, 1850

Kupfer T. et al., 2015, A\&A, 576, A44

Latour M., Fontaine G., Brassard P., Green E. M., Chayer P., Randall S. K., 2011, ApJ, 733, 100

Liebert J., Saffer R. A., Green E. M., 1994, AJ, 107, 1408

Lisker T., Heber U., Napiwotzki R., Christlieb N., Han Z., Homeier D., Reimers D., 2005, A\&A, 430, 223

Lutz R., Schuh S., Silvotti R., Bernabei S., Dreizler S., Stahn T., Hügelmeyer S. D., 2009, A\&A, 496, 469

McDonald I., Zijlstra A. A., Boyer M. L., 2012, MNRAS, 427, 343

McQuillin R., Evans A., Wilson D., Maxted P. F. L., Pollacco D., West R. G., Hounsell R. A., Bode M. F., 2012, MNRAS, 419, 330

Maxted P. F. L., Heber U., Marsh T. R., North R. C., 2001, MNRAS, 326, 1391

Maxted P. F. L., Marsh T. R., Heber U., Morales-Rueda L., North R. C., Lawson W. A., 2002, MNRAS, 333, 231

Maxted P. F. L., Norton A. J., West R. G., Copperwheat C., Marsh T. R., 2008, in Heber U., Jeffery C. S., Napiwotzki R., eds, ASP Conf. Ser. Vol. 392, Hot Subdwarf Stars and Related Objects. Astron. Soc. Pac., San Francisco, p. 179

Mengel J. G., Norris J., Gross P. G., 1976, ApJ, 204, 488

Miller Bertolami M. M., Córsico A. H., Althaus L. G., 2011, ApJ, 741, L3

Morales-Rueda L., Maxted P. F. L., Marsh T. R., North R. C., Heber U., 2003, MNRAS, 338, 752

Naslim N., Jeffery C. S., Behara N. T., Hibbert A., 2011, MNRAS, 412, 363

Németh P., Kawka A., Vennes S., 2012, MNRAS, 427, 2180

Norton A. J. et al., 2011, A\&A, 528, A90

O’Donoghue D., Gray A. E., Kilkenny D., Stobie R. S., Koen C., 1997, MNRAS, 285, 657

O’Donoghue D. et al., 1998a, MNRAS, 296, 296

O’Donoghue D., Koen C., Gray A. E., Kilkenny D., van Wyk F., 1998b, MNRAS, 296, 306

Oreiro R., Pérez Hernández F., Østensen R., Solheim J. E., MacDonald J., Ulla A., 2007, A\&A, 461, 585

Oreiro R., Pérez Hernández F., Ulla A., Garrido R., Østensen R., MacDonald J., 2005, A\&A, 438, 257

Oreiro R., Ulla A., Pérez Hernández F., Østensen R., Rodríguez López C., MacDonald J., 2004, A\&A, 418, 243

Oreiro R., Østensen R. H., Green E. M., Geier S., 2009, A\&A, 496, 827

Østensen R. H., 2010, Astron. Nachr., 331, 1026

Østensen R., Solheim J.-E., Heber U., Silvotti R., Dreizler S., Edelmann H., 2001a, A\&A, 368, 175

Østensen R., Heber U., Silvotti R., Solheim J.-E., Dreizler S., Edelmann H., 2001b, A\&A, 378, 466

Østensen R. H. et al., 2010a, MNRAS, 408, L51

Østensen R. H. et al., 2010b, MNRAS, 409, 1470

Østensen R. H. et al., 2010c, A\&A, 513, A6

Østensen R. H. et al., 2011a, MNRAS, 414, 2860

Østensen R. H. et al., 2011b, ApJ, 731, L13

Østensen R. H. et al., 2012, ApJ, 753, L17

Østensen R. H. et al., 2013, A\&A, 559, A35 
Østensen R. H., Reed M. D., Baran A. S., Telting J. H., 2014a, A\&A, 564, L14

Østensen R. H., Telting J. H., Reed M. D., Baran A. S., Nemeth P., Kiaeerad F., 2014b, A\&A, 569, A15

Pablo H., Kawaler S. D., Green E. M., 2011, ApJ, 740, L47

Pakštiene E., Qvam J. K. T., Østensen R. H., Telting J. H., 2014, Acta Astron., 64, 349

Piccioni A. et al., 2000, A\&A, 354, L13

Pollacco D. L. et al., 2006, PASP, 118, 1407

Ramsay G., Hakala P., 2005, MNRAS, 360, 314

Ramsay G., Napiwotzki R., Hakala P., Lehto H., 2006, MNRAS, 371, 957

Randall S. et al., 2004, Ap\&SS, 291, 465

Randall S. K. et al., 2005, ApJ, 633, 460

Randall S. K. et al., 2006a, ApJ, 643, 1198

Randall S. K. et al., 2006b, ApJ, 645, 1464

Randall S. K., Fontaine G., Charpinet S., Gray A. E., Lopes I. P., O’Toole S. J., Brassard P., 2006c, ApJ, 648, 637

Randall S. K. et al., 2007, A\&A, 476, 1317

Randall S. K., Calamida A., Bono G., 2009, A\&A, 494, 1053

Randall S. K., Calamida A., Bono G., 2010, Ap\&SS, 329, 55

Randall S. K., Calamida A., Fontaine G., Bono G., Brassard P., 2011, ApJ, 737, L27

Randall S. K., Bagnulo S., Ziegerer E., Geier S., Fontaine G., 2015, A\&A, 576, A65

Randall S. K. et al., 2016, A\&A, 589, A1

Reed M. D. et al., 2004a, MNRAS, 348, 1164

Reed M. D. et al., 2004b, ApJ, 607, 445

Reed M. D., Eggen J. R., Zhou A.-Y., Terndrup D. M., Harms S. L., An D., Hashier M. A., 2006, MNRAS, 369, 1529

Reed M. D., Terndrup D. M., Zhou A.-Y., Unterborn C. T., An D., Eggen J. R., 2007a, MNRAS, 378, 1049

Reed M. D. et al., 2007b, ApJ, 664, 518

Reed M. D. et al., 2009, A\&A, 493, 175

Reed M. D. et al., 2010a, Ap\&SS, 329, 83

Reed M. D. et al., 2010b, MNRAS, 409, 1496

Reed M. D. et al., 2011a, MNRAS, 414, 2885

Reed M. D. et al., 2011b, MNRAS, 412, 371

Reed M. D. et al., 2012a, MNRAS, 421, 181

Reed M. D., Baran A., Østensen R. H., Telting J., O’Toole S. J., 2012b, MNRAS, 427, 1245

Reed M. D., Foster H., Telting J. H., Østensen R. H., Farris L. H., Oreiro R., Baran A. S., 2014, MNRAS, 440, 3809

Reed M. D. et al., 2016, MNRAS, 458, 1417

Ricker G. R. et al., 2015, J. Astron. Telescopes Instrum. Syst., 1, 014003

Rodríguez-López C. et al., 2010, MNRAS, 401, 23

Saffer R. A., Bergeron P., Koester D., Liebert J., 1994, ApJ, 432, 351

Schaffenroth V., Barlow B. N., Drechsel H., Dunlap B. H., 2015, A\&A, 576, A123

Schuh S., Dreizler S., Deetjen J. L., Heber U., Geckeler R. D., 2000, Balt. Astron. 9, 395
Schuh S., Huber J., Dreizler S., Heber U., O'Toole S. J., Green E. M., Fontaine G., 2006, A\&A, 445, L31

Silvotti R., Solheim J.-E., Gonzalez Perez J. M., Heber U., Dreizler S., Edelmann H., Østensen R., Kotak R., 2000, A\&A, 359, 1068

Silvotti R., Østensen R., Heber U., Solheim J.-E., Dreizler S., Altmann M., 2002a, A\&A, 383, 239

Silvotti R. et al., 2002b, A\&A, 389, 180

Silvotti R. et al., 2006, A\&A, 459, 557

Silvotti R., Randall S. K., Dhillon V. S., Marsh T. R., Savoury C. D., Schuh S., Fontaine G., Brassard P., 2010, Astron. Nachr., 331, 1034

Smalley B., Smith K. C., Dworetsky M. M., 2001, UCLSYN Userguide. Available at: http://www.astro.keele.ac.uk/bs/uclsyn.pdf

Smalley B. et al., 2011, A\&A, 535, A3

Smalley B. et al., 2014, A\&A, 564, A69

Smalley B. et al., 2017, MNRAS, 465, 2662

Solheim J.-E., Østensen R., 2006, Balt. Astron., 15, 231

Solheim J.-E., Østensen R., Silvotti R., Heber U., 2004, Ap\&SS, 291, 419

Stobie R. S., Kawaler S. D., Kilkenny D., O’Donoghue D., Koen C., 1997a, MNRAS, 285, 651

Stobie R. S. et al., 1997b, MNRAS, 287, 848

Tamuz O., Mazeh T., Zucker S., 2005, MNRAS, 356, 1466

Telting J. H., Geier S., Østensen R. H., Heber U., Glowienka L., Nielsen T., Oreiro R., Frandsen S., 2008, A\&A, 492, 815

Telting J. H. et al., 2012, A\&A, 544, A1

Telting J. H. et al., 2014, A\&A, 570, A129

Thomas N. L., Norton A. J., Pollacco D., West R. G., Wheatley P. J., Enoch B., Clarkson W. I., 2010, A\&A, 514, A30

Turner O., Anderson D. R., Maxted P. L. F., Hellier C., 2015, European Planetary Science Congress 2015, 10, EPSC2015. Available at: http://meetingorganizer.copernicus.org/EPSC2015

Van Grootel V., Charpinet S., Fontaine G., Green E. M., Brassard P., 2010a, A\&A, 524, A63

Van Grootel V. et al., 2010b, ApJ, 718, L97

Vennes S., Kawka A., Németh P., 2011, MNRAS, 410, 2095

Viton M., Deleuil M., Tobin W., Prevot L., Bouchet P., 1991, A\&A, 242, 175

Vučković M. et al., 2006, ApJ, 646, 1230

Vučković M., Aerts C., Östensen R., Nelemans G., Hu H., Jeffery C. S., Dhillon V. S., Marsh T. R., 2007, A\&A, 471, 605

Vučković M., Østensen R. H., Acke B., Reed M. D., Oreiro R., Bloemen S., Baran A. S., 2012, in Kilkenny D., Jeffery C. S., Koen C., eds, ASP Conf. Ser. Vol. 452, Fifth Meeting on Hot Subdwarf Stars and Related Objects. Astron. Soc. Pac., San Francisco, p. 257

Webbink R. F., 1984, ApJ, 277, 355

Woudt P. A. et al., 2006, MNRAS, 371, 1497

Zhou A.-Y. et al., 2006, MNRAS, 367, 179

This paper has been typeset from a $\mathrm{T}_{\mathrm{E}} \mathrm{X} / \mathrm{L} \mathrm{T}_{\mathrm{E}} \mathrm{X}$ file prepared by the author. 